\title{
Atuação do enfermeiro na identificação dos fatores associados à não adesão ao idoso no tratamento de hipertensão arterial sistêmica
}

\author{
Nurse's performance in the identification of factors associated with non-adhesion to the elderly in \\ the treatment of systemic arterial hypertension \\ Desempeño de la enfermera en la identificación de factores asociados a la no adhesión a ancianos en \\ el tratamiento de la hipertensión arterial sistémica
}

Recebido: 11/01/2021 | Revisado: 14/01/2021 | Aceito: 22/01/2021 | Publicado: 25/01/2021

Cintia Hellen Souto Ramos

ORCID: https://orcid.org/0000-0003-1061-0921

E-mail Cintiahellen2007@ hotmail.com

Assunção Gomes Adeodato

ORCID: https://orcid.org/0000-0002-5312-9815

Faculdade de Terra Nordeste, Brasil

E-mail: assuncaoadeodato@gmail.com

Nicolau da Costa

ORCID: http://orcid.org/0000-0001-9845-7292

Faculdade de Terra Nordeste, Brasil

E-mail:nickddacosta@gmail.com

Diego Jorge Maia Lima

ORCID: http://orcid.org/0000-0003-4708-3088

Faculdade de Terra Nordeste, Brasil

E-mail: diegojorge19@hotmail.com

Jéssica Luzia Delfino Pereira

ORCID: https://orcid.org/0000-0002-4940-6525

Escola de Saúde Pública Ceará,Brasil

E-mail: enf.jessicaluzia@gmail.com

Nicely Alexandra da Silva

ORCID: https://orcid.org/0000-0001-7050-7163

Centro Universitário Unifametro, Brasil

E-mail: nicelyalexandra@hotmail.com

\begin{abstract}
Resumo
Presente estudo tem por finalidade descrever atuação do enfermeiro na identificação dos fatores associados à não adesão ao idoso no tratamento de hipertensão arterial sistêmica. Metodologia trata-se de revisão integrativa. Para seleção dos estudos foram utilizadas quatro bases de dados, a saber: Literatura Latino Americana e do Caribe em Ciência de Saúde (LILACS), Literatura Internacional em Ciências da Saúde (MEDLINE), (PUBMED) e Allied Health Literature (CINAHL). A busca foi realizada no mês de novembro de 2020.Para a seleção dos artigos foram considerados os seguintes critérios de inclusão artigos completos, disponíveis eletronicamente. Foram selecionados 13 artigos que atenderam os objetivos proposto Resultados: foi possível observar os fatores preditoras para adesão medicamentosa para o tratamento da HAS em idoso se dá pela falta de informações, baixa escolaridade, falta de apoia familiar. Considerações finais: faz-se necessário que os enfermeiros reconheçam os principais fatores que contribuem para a não adesão ao tratamento medicamentoso ao idoso. Portanto a necessidade de criar uma estratégia que envolvam acolhimento, educação em saúde e mudança de comportamento podem favorecer um comportamento participativo que contribua para a adesão terapêutica de maneira adequada.
\end{abstract}

Palavras-chave: Hipertensão arterial; Não adesão ao medicamento; Enfermagem; Atenção integral ao idoso.

\begin{abstract}
This study aims to describe the role of nurses in identifying factors associated with non-adherence to the elderly in the treatment of systemic arterial hypertension. Methodology is an integrative review. To select the studies, four databases were used, namely: Latin American and Caribbean Literature in Health Science (LILACS), International Literature in Health Sciences (MEDLINE), (PUBMED) and Allied Health Literature (CINAHL). The search was carried out in November 2020. For the selection of articles, the following inclusion criteria were considered complete articles, available electronically. 13 articles were selected that met the proposed objectives Results: it was possible to observe the predictive factors for medication adherence for the treatment of SAH in the elderly, due to the lack of information, low education, lack of family support. Final considerations: it is necessary for nurses to recognize the
\end{abstract}


main factors that contribute to non-adherence to medication treatment for the elderly. Therefore, the need to create a strategy that involves embracement, health education and behavior change can favor participatory behavior that contributes to therapeutic adherence in an appropriate manner.

Keywords: Arterial hypertension; Non-adherence to the medication; Nursing; Comprehensive care for the elderly.

\section{Resumen}

Este estudio tiene como objetivo describir el papel de las enfermeras en la identificación de factores asociados a la falta de adherencia a los ancianos en el tratamiento de la hipertensión arterial sistémica. La metodología es una revisión integradora. Para seleccionar los estudios se utilizaron cuatro bases de datos, a saber: Literatura Latinoamericana y del Caribe en Ciencias de la Salud (LILACS), Literatura Internacional en Ciencias de la Salud (MEDLINE), (PUBMED) y Literatura en Salud Aliada (CINAHL). La búsqueda se realizó en noviembre de 2020. Para la selección de artículos se consideraron los siguientes criterios de inclusión artículos completos, disponibles electrónicamente. Se seleccionaron 13 artículos que cumplieron con los objetivos propuestos. Resultados: fue posible observar los factores predictores de adherencia a la medicación para el tratamiento de la HAS en el anciano, debido a la falta de información, baja educación, falta de apoyo familiar. Consideraciones finales: es necesario que el enfermero reconozca los principales factores que contribuyen a la no adherencia al tratamiento farmacológico del anciano. Por lo tanto, la necesidad de crear una estrategia que involucre acogida, educación para la salud y cambio de conducta puede favorecer un comportamiento participativo que contribuya a la adherencia terapéutica de manera adecuada.

Palabras clave: Hipertensión arterial; No adherencia a la medicación; Enfermería; Atención integral a la tercera edad.

\section{Introdução}

No Brasil, principalmente nas últimas décadas vem ocorrendo ampla mobilização para implementação de estratégias para prevenção, adesão ao tratamento e controle das doenças crônicas por meio da reorganização dos serviços em saúde. Dentre as estratégias, surgiu em 1994, o programa saúde da família (PSF), agora denominado de estratégias de saúde da família (ESF), (Jheilh, 2016).

Observa-se com aumento populacional acarreta são imensos, pois ocorre aumento dos custos com doenças infecciosas, nutricionais e crônicas que necessitam de cuidado contínua da equipe multiprofissionais (Silveira \& Silva et al.,2021).

De acordo com dados da Vigilância de Fatores de Risco e Proteção para Doenças Crônicas por Inquérito Telefônico, a prevalência de HAS é de $24,8 \%$, com variação conforme a faixa etária estudada entre $22,0 \%$ na população acima de 18 anos e $69,9 \%$ nos idosos (Gewehr et al.,2018).

A portaria $\mathrm{n}^{\circ} 483$, de $1^{\circ}$ de abril de 2014 do Ministério da Saúde do Brasil que redefine a Rede de Atenção à Saúde das pessoas com Doenças Crônicas no âmbito do Sistema Único de Saúde (SUS) e estabelece diretrizes para a organização das suas linhas de cuidado, define as doenças crônicas como aquelas que apresentam início gradual, com duração longa ou incerta, que, em geral, apresentam múltiplas causas e cujo tratamento envolve mudanças de estilo de vida, em um processo de cuidado contínuo que, usualmente, não leva a cura.

Assim, com e implementação do programa nacional o Hiperdia que é destinado ao cadastramento e acompanhamento de portadores de hipertensão arterial e/ou diabetes mellitus atendidos na rede ambulatorial do Sistema Único de Saúde - SUS, permitindo gerar informação para aquisição, dispensação e distribuição de medicamentos de forma regular e sistemática a todos os pacientes cadastrados. O sistema envia dados para o Cartão Nacional de Saúde, funcionalidade que garante a identificação única do usuário do Sistema Único de Saúde - SUS (Brasil,2013).

Verifica-se que os desafios da adesão ao tratamento, controle e prevenção da HAS no Brasil são, sobretudo, das equipes das ESF. Os profissionais enfermeiros têm a importância primordial nas estratégias para adesão ao tratamento, prevenção e diagnóstico, monitorização e controle da HAS. Devem também, ter sempre em foco o princípio fundamental da prática centrada na pessoa e, consequentemente, envolver as famílias e cuidadores, em nível individual e coletivo (Torres \& Figueiredo, et al.,2018). 
A adesão ao tratamento de uma doença consiste em seguir o que foi proposto pelos profissionais de saúde. No que se refere ao tratamento farmacológico, a não adesão significa o abandono do uso dos medicamentos, sem orientação médica ou a execução de forma irregular do tratamento, seja na prática de atrasar a tomada do medicamento ou de realizar pequenas interrupções da terapêutica prescrita. A baixa adesão ao tratamento é um dos principais fatores para a persistência de valores elevados da PA (Gewehr, et al.,2018).

De acordo com LEI No 10.741, DE $1^{\circ}$ DE OUTUBRO DE 2003. Sobre estatuto do idoso é assegurada a atenção integral à saúde do idoso, por intermédio do Sistema Único de Saúde - SUS, garantindo-lhe o acesso universal e igualitário, em conjunto articulado e contínuo das ações e serviços, para a prevenção, promoção, proteção e recuperação da saúde, incluindo a atenção especial às doenças que afetam preferencialmente os idosos. A prevenção e a manutenção da saúde do idoso serão efetivadas por meio de: cadastramento da população idosa em base territorial; atendimento geriátrico e gerontológico em ambulatórios; unidades geriátricas de referência, com pessoal especializado nas áreas de geriatria e gerontologia social (Brasil,2003).

O enfermeiro, como integrante da equipe de saúde, assume a corresponsabilidade das ações do cuidado para a promoção da saúde e prevenção de riscos e agravos dessa doença, como no controle e acompanhamento do portador de HAS. Por meio do conhecimento científico e de seu papel de educador, ele tem a possibilidade de instrumentalizar o portador da doença para o tratamento, melhorando sua qualidade de vida (Costa \& Araújo et al.,2014).

Entre as preocupações principais sobre a não adesão ao tratamento da hipertensão arterial no idoso. Quais são atuação do enfermeiro na identificação aos fatores associados à não adesão ao idoso no tratamento de hipertensão arterial sistêmica?

O interesse deste estudo dá se pela necessidade recorrente dos pacientes idosos que não aderiam ao tratamento de hipertensão. Com tal constatação surgiu o interesse de pesquisar atuação do enfermeiro na identificação aos fatores associados à não adesão ao idoso no tratamento de hipertensão arterial sistêmica.

A relevância desse estudo implica em subsidiar o planejamento de ações da atenção básica voltadas ao controle da hipertensão arterial, visto que a prevalência dessas doenças tem se mostrado crescente no Brasil. Assim, a importância da orientação do profissional do enfermeiro no que tange a adesão do idoso ao tratamento farmacológica na atenção básica para a redução de agravos e riscos das doenças cardiovasculares, bem como atuação dos profissionais de saúde na atenção primária na atividade educativa para promover melhor a qualidade de vida desta população vulneráveis.

Assim, o estudo tem como objetivo descrever atuação do enfermeiro na identificação dos fatores associados à não adesão ao idoso no tratamento de hipertensão arterial sistêmica

\section{Metodologia}

Trata-se de uma revisão integrativa, em que se considera uma pesquisa ampla, para propiciar a inclusão de estudos clínicos de maneira sistemática e ordenada, contribuindo para o aprofundamento do conhecimento do tema investigado (Cunha,2014). Para realização da revisão integrativa é necessário seguir as seguintes etapas (Mendes, Silveira \& Galvão 2019):

Primeira etapa foi realizada a identificação do tema ou questionamento da revisão integrativa, consiste na elaboração da questão da pesquisa e do tema delimitado para a construção da revisão integrativa e, posteriormente, para a definição das palavras-chave a ser utilizadas na estratégia de busca dos estudos. Para nortear a pesquisa que se delineia, formulou-se a questão: Para tanto, o estudo busca responder a seguinte problemática: Diante do exposto surgiu o seguinte questionamento: Quais são atuação do enfermeiro na identificação dos fatores associados à não adesão ao idoso no tratamento de hipertensão arterial sistêmica?

Na segunda etapa integrou o estabelecimento de critérios para inclusão e exclusão de estudos e amostragem da busca na literatura. Assim foi realizada a busca nas bases de dados para identificar os estudos a serem incluídos ou excluídos da 
pesquisa. Foram adotados os seguintes critérios de inclusão: ser artigo elegível publicado na íntegra, estar disponível online, escrito em português, inglês ou espanhol e que apresenta o percurso metodológico que permita alcançar os objetivos proposta. Antes de aplicar o instrumento da pesquisa foi realizada uma leitura, para que pudesse excluir os artigos que não se adequassem ao objetivo do estudo, como também excluir duplicidade dos artigos com a mesma base.

Em seguida, foi realizada busca por artigos indexados na base de dados Bireme utilizando os Descritores em Ciências da Saúde (DeCS) e operador booleano AND, formando, assim, a estratégia de busca a partir dos descritores e termos controlados, com a seguinte combinação: Hipertensão arterial AND Não adesão ao Medicamento AND Enfermagem AND Atenção Integral ao Idoso. Para seleção dos estudos foram utilizadas quatro bases de dados, a saber: Literatura Latino Americana e do Caribe em Ciência de Saúde (LILACS), Literatura Internacional em Ciências da Saúde (MEDLINE), (PUBMED) e Allied Health Literature (CINAHL). A busca foi realizada no mês de novembro de 2020

A busca nas bases de dados resultou na identificação de 130 artigos. Foram excluídos os artigos por não se adequar com objetivo proposto, por repetição e por não obedecer aos critérios de inclusão, por duplicidade e por não responder à pergunta norteadora. Após a leitura final foram selecionados 13 artigos para serem discutidos no desenvolvimento desse trabalho.

Figura 1. Fluxograma Prisma: caracterização do número de artigos pesquisados nas bases de dados: LILACS, MEDLINE, PUBMED, CINAHL. Caucaia-Ceará, novembro, 2020.
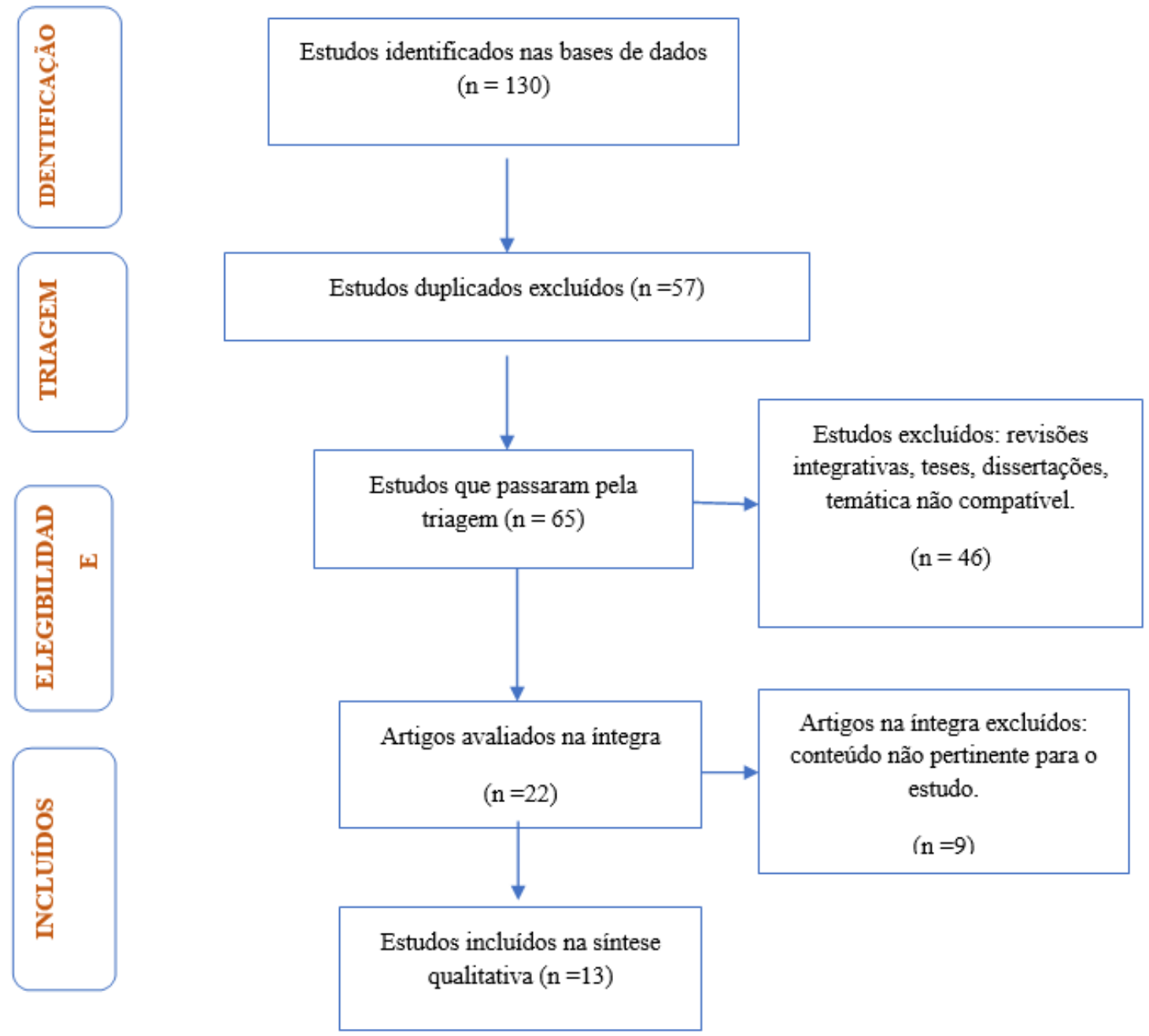

Fonte: Autores. 
A terceira etapa foi utilizada um instrumento de coleta de dados, que tem como objetivo extrair informação-chave de cada artigo selecionado, por meio de instrumento que possa reunir e sintetizar os dados relevantes individualmente de maneira compreensível.

Quarta etapa foi realizada a apresentação dos resultados e discussão dos dados foi feita de forma descritiva, cujo método possibilita a avaliação da aplicabilidade deste estudo, de forma a atingir o objetivo desta revisão integrativa.

Quinta etapa é a interpretação dos resultados é análoga à discussão de resultados em estudos primários. Assim foi realizada a comparação dos dados evidenciados nos artigos incluídos na revisão integrativa com o conhecimento teórico. Em seguida foi feito a discussão dos resultados pertinentes de maneira de comparativa com o conhecimento teórico que é fundamentado nos resultados da avaliação crítica dos estudos incluídos na pesquisa. Desta forma identifica conclusões e implicações resultantes da revisão integrativa, bem como lacunas que permitam ser apontadas como sugestões para as evidências futuras.

Sexta etapa foi realizada a apresentação da revisão integrativa que está disposta no item de resultados e discussões. Nesta fase optou-se por elaborar um quadro que classificassem os artigos por cores utilizados na revisão, onde o mesmo faz menção a metodologia e objetivo dos estudos.

\section{Resultados e Discussão}

O quadro a seguir demonstra informações dos artigos selecionados como autores, título dos artigos, objetivos, revistas, ano de publicação, resultados.

Quadro 1: Caracterização dos artigos quanto número do artigo; título do estudo; autores/ano; objetivos; resultados. CaucaiaCeará novembro,2020

\begin{tabular}{|c|c|c|c|c|c|c|}
\hline $\mathbf{N}^{\mathbf{0}}$ & Autor(res) & Título do estudo & Objetivo do estudo & Revista & Síntese dos resultados & Ano \\
\hline A1 & $\begin{array}{l}\text { Silva, M } \\
\text { OM.et al., }\end{array}$ & $\begin{array}{c}\text { Acompanhamento } \\
\text { farmacêutico: adesão e } \\
\text { problemas relacionados à } \\
\text { farmacoterapia de idosos }\end{array}$ & $\begin{array}{l}\text { realizar uma análise da adesão } \\
\text { farmacoterapia, presença de } \\
\text { poli farmácia e possíveis } \\
\text { problemas relacionados a } \\
\text { medicamentos nos idosos }\end{array}$ & $\begin{array}{l}\text { Journal of } \\
\text { Biology \& } \\
\text { Pharmacyand } \\
\text { Agricultural } \\
\text { Managemen }\end{array}$ & $\begin{array}{l}\text { foram catalogados } 33 \text { medicamentos e } \\
\text { identificados } 15 \text { problemas a eles } \\
\text { relacionados, estando presente em } \\
60,0 \% \text { dos sujeitos. Observou-se } \\
\text { que } 30,0 \% \text { desses idosos são } \\
\text { polimedicados. Dessa forma, foram } \\
\text { realizados um total de } 16 \\
\text { intervenções, por meio de ações } \\
\text { educativas e orientações sobre o } \\
\text { regime terapêutico }\end{array}$ & 2020 \\
\hline A2 & $\begin{array}{c}\text { Gauterio } \\
\text { Abreu et al., }\end{array}$ & $\begin{array}{l}\text { Fatores comportamentais } \\
\text { associados à adesão } \\
\text { medicamentosa em idosos } \\
\text { em atendimento } \\
\text { ambulatorial }\end{array}$ & $\begin{array}{l}\text { avaliar a relação entre fatores } \\
\text { comportamentais e adesão à } \\
\text { terapêutica medicamentosa } \\
\text { em idosos em atendimento } \\
\text { ambulatoria }\end{array}$ & $\begin{array}{c}\text { Revista de } \\
\text { Enfermagem } \\
\text { do Centro-Oeste } \\
\text { Mineiro }\end{array}$ & $\begin{array}{l}\text { s idosos que não acreditavam na } \\
\text { importância do uso dos } \\
\text { medicamentos, não sabiam o nome } \\
\text { destes, usavam-nos somente na } \\
\text { quando apresentavam sintomas, } \\
\text { esqueciam-se de utilizá-los ou não } \\
\text { seguiam a prescrição conforme a } \\
\text { indicação médica, tinham menor } \\
\text { mediana de adesão do que os que não } \\
\text { apresentavam esses comportamentos. }\end{array}$ & 2019 \\
\hline A3 & $\begin{array}{l}\text { Falcão AS, } \\
\text { Silva MGC, } \\
\text { et al. }\end{array}$ & $\begin{array}{l}\text { Estilo de vida e adesão ao } \\
\text { tratamento de hipertensão } \\
\text { artéria Sistêmica em } \\
\text { homens idosos }\end{array}$ & $\begin{array}{l}\text { Avaliar estilo de vida e adesão } \\
\text { ao tratamento de hipertensão } \\
\text { arterial sistêmica (HAS) em } \\
\text { homens idosos }\end{array}$ & $\begin{array}{l}\text { Rev Bras } \\
\text { Promoç Saúde, } \\
\text { Fortaleza }\end{array}$ & $\begin{array}{l}\text { Constatou-se a adesão parcial dos } \\
\text { homens idosos avaliados, os quais } \\
\text { utilizam alguns hábitos saudáveis } \\
\text { como mecanismo compensatório para } \\
\text { manter práticas que dificultam a } \\
\text { adesão ao tratamento. }\end{array}$ & 2018 \\
\hline
\end{tabular}




\begin{tabular}{|c|c|c|c|c|c|c|}
\hline A4 & $\begin{array}{l}\text { Gewehr } \\
\text { DM, } \\
\text { Bandeira } \\
\text { VAC et al., }\end{array}$ & $\begin{array}{l}\text { Adesão ao tratamento } \\
\text { farmacológico da } \\
\text { hipertensão arterial na } \\
\text { Atenção Primária à Saúde }\end{array}$ & $\begin{array}{l}\text { Verificar a adesão ao } \\
\text { tratamento farmacológico da } \\
\text { hipertensão arterial e fatores } \\
\text { associados à baixa adesão em } \\
\text { hipertensos adstritos à } \\
\text { Atenção Primária à Saúde. }\end{array}$ & Saúde debate & $\begin{array}{l}\text { Os fatores relacionados com a } \\
\text { diminuição da adesão foram: baixa } \\
\text { renda, uso de dois ou mais } \\
\text { antihipertensivos e dificuldades para } \\
\text { ler a embalagem dos medicamentos }\end{array}$ & 2018 \\
\hline A5 & $\begin{array}{c}\text { Pinheiro } \\
\text { FM, } \\
\text { Espirito } \\
\text { Santo FH, et } \\
\text { al }\end{array}$ & $\begin{array}{l}\text { Adesão Terapêutica Em } \\
\text { Idosos Hipertensos: } \\
\text { Revisão Integrativa }\end{array}$ & $\begin{array}{l}\text { Identificar evidências sobre } \\
\text { adesão terapêutica em idosos } \\
\text { hipertensos }\end{array}$ & $\begin{array}{l}\text { Revista de } \\
\text { Enfermagem do } \\
\text { Centro-Oeste } \\
\text { Mineiro }\end{array}$ & $\begin{array}{l}\text { Identificou-se que as evidências sobre } \\
\text { adesão terapêutica em idosos } \\
\text { hipertensos, estão relacionadas, ao } \\
\text { Desenvolvimento das doenças } \\
\text { crônicas não transmissíveis e ao estilo } \\
\text { de vida da pessoa idosa. Estratégias } \\
\text { que envolvam atitudes e } \\
\text { Comportamentos dos idosos podem } \\
\text { favorecer um comportamento } \\
\text { participativo que contribua para uma } \\
\text { adequada adesão Terapêutica. }\end{array}$ & 2018 \\
\hline A6 & $\begin{array}{l}\text { Vasconcelos } \\
\text { Thais et al., }\end{array}$ & $\begin{array}{l}\text { Fatores associados à não } \\
\text { adesão ao tratamento de } \\
\text { pacientes com hipertensão } \\
\text { arterial sistêmica: uma } \\
\text { revisão integrativa da } \\
\text { literatura }\end{array}$ & $\begin{array}{c}\text { Analisar na produção } \\
\text { cientifica quais os fatores } \\
\text { associados, a não adesão ao } \\
\text { tratamento da hipertensão } \\
\text { arterial }\end{array}$ & $\begin{array}{l}\text { Ciências } \\
\text { Biológicas e de } \\
\text { Saúde Unit }\end{array}$ & $\begin{array}{l}\text { Dentre esses, observa-se que baixa } \\
\text { escolaridade, menor idade, nível } \\
\text { socioeconômico baixo, etilismo, curto } \\
\text { tempo de diagnóstico da doença, } \\
\text { baixo grau de conhecimento sobre a } \\
\text { doença e maior número de } \\
\text { medicamentos usados são fatores } \\
\text { significantes para a não adesão. }\end{array}$ & 2017 \\
\hline A7 & $\begin{array}{l}\text { Ribeiro, Í } \\
\text { AP }\end{array}$ & $\begin{array}{c}\text { Tratamento } \\
\text { Farmacológico E Não } \\
\text { Farmacológico De Idosos } \\
\text { Com Hipertensão Arterial: } \\
\text { Adesão Às Terapias } \\
\text { Propostas }\end{array}$ & $\begin{array}{l}\text { Identificar e analisar como } \\
\text { ocorre o processo de adesão } \\
\text { às terapias farmacológicas e } \\
\text { não farmacológicas por } \\
\text { idosos hipertensivos. }\end{array}$ & Revista Uningá & $\begin{array}{l}\text { Há falta de adesão e compreensão } \\
\text { sobre a adequabilidade terapêutica } \\
\text { anti-hipertensiva, devido grande parte } \\
\text { dos idosos serem semianalfabetos de } \\
\text { baixo poder socioeconômico, com } \\
\text { comorbidades, em terapia múltipla } \\
\text { farmacológica e pode apresentar } \\
\text { senilidade, fatores estes que } \\
\text { dificultam a adesão medicamentosa. }\end{array}$ & 2017 \\
\hline A8 & $\begin{array}{c}\text { Tavares } \\
\text { DMS, et al. }\end{array}$ & $\begin{array}{l}\text { Qualidade de vida e } \\
\text { adesão ao tratamento } \\
\text { farmacológico entre } \\
\text { idosos hipertensos }\end{array}$ & $\begin{array}{l}\text { Comparar as variáveis obtidas } \\
\text { em situação de adesão e não } \\
\text { adesão ao tratamento } \\
\text { farmacológico para } \\
\text { hipertensão arterial sistêmica, } \\
\text { segundo condições } \\
\text { socioeconômicas, tempo de } \\
\text { diagnóstico, morbidades autor } \\
\text { referidas, indicativo de } \\
\text { depressão e } \\
\text { Qualidade de vida. }\end{array}$ & $\begin{array}{l}\text { Rev Bras } \\
\text { Enferm }\end{array}$ & $\begin{array}{c}\text { Devem-se preconizar ações que } \\
\text { favoreçam a maior adesão ao } \\
\text { tratamento farmacológico, visando } \\
\text { minimizar o impacto das } \\
\text { comorbidades e melhorar a qualidade } \\
\text { de vida }\end{array}$ & 2016 \\
\hline A9 & $\begin{array}{l}\text { Silva de } \\
\text { Jesus et al., }\end{array}$ & $\begin{array}{c}\text { Adesão ao Tratamento e } \\
\text { Controle da Pressão } \\
\text { Arterial Após Participação } \\
\text { no Rehot }\end{array}$ & $\begin{array}{l}\text { Verificar a adesão ao } \\
\text { tratamento e fatores } \\
\text { associados, bem como o } \\
\text { controle da pressão arterial } \\
\text { (PA) em participantes de o } \\
\text { ensaio clínico do tratamento } \\
\text { óptimo da hipertensão } \\
\text { resistente (ReHOT) }\end{array}$ & $\begin{array}{l}\text { Sociedade } \\
\text { Brasileira de } \\
\text { Cardiologia }\end{array}$ & $\begin{array}{l}\text { Notamos que tanto o tratamento } \\
\text { quanto a adesão a ele e o controle da } \\
\text { PA são de extrema importância para o } \\
\text { diagnóstico correto da HAS }\end{array}$ & 2016 \\
\hline A10 & $\begin{array}{l}\text { Marin NS, } \\
\text { Santos MF, } \\
\text { Moro AS }\end{array}$ & $\begin{array}{l}\text { Percepção de hipertensos } \\
\text { sobre a sua não adesão ao } \\
\text { uso de medicamentos }\end{array}$ & $\begin{array}{l}\text { Analisar a percepção dos } \\
\text { portadores de HA sobre a não } \\
\text { adesão ao uso de } \\
\text { medicamentos }\end{array}$ & $\begin{array}{c}\text { Rev Esc Enferm } \\
\text { US }\end{array}$ & $\begin{array}{c}\text { As complexas condições que } \\
\text { envolvem a não adesão ao tratamento } \\
\text { e ao o atual contexto de predomínio } \\
\text { de doenças crônicas, é primordial que } \\
\text { se invista em estratégias inovadoras } \\
\text { de cuidado a tais pessoas }\end{array}$ & 2016 \\
\hline A11 & $\begin{array}{l}\text { Magnabosc } \\
\text { o P, et al., }\end{array}$ & $\begin{array}{l}\text { Análise comparativa da } \\
\text { não adesão ao tratamento }\end{array}$ & $\begin{array}{l}\text { Avaliar os índices e os } \\
\text { principais fatores associados à }\end{array}$ & $\begin{array}{l}\text { Rev. } \\
\text { Latino.America }\end{array}$ & $\begin{array}{l}\text { As características sociodemográficas, } \\
\text { econômicas, hábitos de vida e o modo }\end{array}$ & 2015 \\
\hline
\end{tabular}




\begin{tabular}{|c|c|c|c|c|c|c|}
\hline & & $\begin{array}{c}\text { medicamentoso da } \\
\text { Hipertensão arterial } \\
\text { sistêmica em população } \\
\text { urbana e rural }\end{array}$ & $\begin{array}{l}\text { não adesão ao tratamento } \\
\text { medicamentoso da } \\
\text { hipertensão arterial sistêmica, } \\
\text { entre área urbana e rural }\end{array}$ & $\begin{array}{l}\text { na de Enferma- } \\
\text { gem }\end{array}$ & $\begin{array}{l}\text { de relacionar-se com os serviços de } \\
\text { saúde foram os fatores que } \\
\text { apresentaram associaçãa com a não } \\
\text { adesão, independentemente do local } \\
\text { de residência }\end{array}$ & \\
\hline A12 & $\begin{array}{l}\text { Barreto Ms } \\
\quad \text { et al., }\end{array}$ & $\begin{array}{l}\text { Conhecimento sobre } \\
\text { hipertensão arterial e } \\
\text { fatores associados à não } \\
\text { adesão à farmacoterapia }\end{array}$ & $\begin{array}{c}\text { Identificar o nível de } \\
\text { conhecimento de pessoas com } \\
\text { hipertensão arterial acerca da } \\
\text { doença e verificar os fatores } \\
\text { associados à não adesão à } \\
\text { farmacoterapia anti- } \\
\text { hipertensiva }\end{array}$ & $\begin{array}{l}\text { Rev. } \\
\text { Latino.America } \\
\text { na de Enferma- } \\
\text { gem }\end{array}$ & $\begin{array}{l}\text { Conhecimento sobre a doença e } \\
\text { insatisfação com o serviço de saúde } \\
\text { influenciam no processo de não } \\
\text { adesão ao tratamento medicamentoso } \\
\text { anti-hipertensivo }\end{array}$ & 2014 \\
\hline A13 & $\begin{array}{l}\text { Barreto MS, } \\
\text { et al. }\end{array}$ & $\begin{array}{l}\text { Prevalência de não adesão } \\
\text { à Farmacoterapia Anti- } \\
\text { hipertensiva } \\
\text { e fatores associados }\end{array}$ & $\begin{array}{l}\text { Verificar a prevalência da não } \\
\text { adesão ao tratamento } \\
\text { medicamentoso anti- } \\
\text { hipertensivo e sua associação } \\
\text { com fatores biosócio- } \\
\text { econômicos e assistenciais }\end{array}$ & $\begin{array}{c}\text { Revista } \\
\text { Brasileira de } \\
\text { Enfermagem }\end{array}$ & $\begin{array}{c}\text { dificuldade de acesso ao serviço } \\
\text { necessitam de intervenções } \\
\text { diferenciadas, a fim de estimulá-los a } \\
\text { aderirem ao tratamento } \\
\text { medicamentoso }\end{array}$ & 2014 \\
\hline
\end{tabular}

Fonte: Elaborada pelos autores com dados da pesquisa (2020).

Em relação a síntese dos resultados encontrados nos estudos mostram a falta de informações, baixa escolaridade, falta de apoia familiar, atendimento dos profissionais. falta das informações pode influência nos fatores a não adesão ao tratamento HAS. Um estudo realizado por Jesus \& Nogueira et al., (2016), avaliando a taxa de adesão medicamentosa, utilizando o Escala de Adesão Terapêutica de Morisky (MMAS), houve diferença de adesão de acordo com a escolaridade dos pacientes. Evidenciou-se que os que apresentavam até nove anos de escolaridade aderiram menos ao tratamento medicamentoso, o que não ocorreu naqueles com mais de nove anos de escolaridade, com a taxa de não adesão medicamentosa mostrou bastante variável nos estudos selecionados, variando de 31,3\% (Jesus \& Nogueira et al., 2016).

Nota-se que maiorias das populações são idosos e que tem algumas limitações no que tange ao uso de medicamento e na captação das orientações dos profissionais enfermagem. Já no estudo realizado por Magnabosco et al., (2015) mostrou prevalência de $61 \%$ Uma das hipóteses que pode justificar a discrepância dos resultados poderia ser os diferentes métodos utilizados e as diferentes populações estudadas. Verificou-se que as experiências e práticas culturais são fatores que influencia ao tratamento da hipertensão arterial sistêmica também os maus hábitos, e a falta de informação e o bom acolhimento de alguns profissionais interferem na adesão ao tratamento dos hipertensos idosos.

Corroborando com o achado Jannuzzi et al., (2014) em seu estudo realizado no ambulatório geral de adultos de um hospital universitário de grande porte no interior do estado de São Paulo também encontrou essa relação. As crenças positivas a respeito dos medicamentos podem estar relacionadas à percepção dos benefícios destes, à medida que os idosos os utilizam e têm melhora em seu quadro de saúde (Barba et al.,2018). E fatores culturais são um dos aspectos mencionados pelos autores como também apoio das famílias orientação destes idosos que muitas das vezes tem dificuldade de ler ou lembrar dos horários da medicação.

Verifica-se que a Sociedade Brasileira de Hipertensão,(2014).afirmaram que uma das explicação para adesão ao tratamento é a falta de informação o despreparo de alguns profissionais de saúde, a demora das marcações das consultas, a falta de acompanhamento da família, torna essa situação vulnerável para mais ocorrências e complicações causadas pela pressão arterial elevada hipertensão arterial sistêmica (HAS), pesquisas comprovam que na idade mais avançada há maior probabilidade de ocorrer o não tratamento para hipertensão arterial no idoso. Principalmente em idosos com mais de 60 anos.

Diante disso, é importante considerar que, independentemente da idade, a adesão ao tratamento para hipertensão arterial sistêmica (HAS) é um processo contínuo que necessita de adaptações, requer da família do companheiro, dos 
profissionais de saúde envolvida nesse processo ofereça ajuda, que investigue de modo a intervi nos problemas detectados e assim melhorar a adesão ao tratamento e assim controlar a hipertensão arterial dessa população (Bezerra, et al.,2014).

Adesão em idosos hipertensos, $42 \%$ desconheciam o caráter crônico da hipertensão e acreditavam na cura da hipertensão essencial e 20\% não sabiam que a hipertensão aumenta o risco de morbimortalidade cardiovascular (Pinheiro et al., 2018).

Portanto, o papel do enfermeiro torna-se imperativo para que o compartilhamento dos conhecimentos seja eficaz e para que ambos, enfermeiro e portador, consigam dominar o conhecimento sobre a doença e a realidade do portador, a fim de estabelecerem, juntos, condutas precisas para atraem os pacientes (Costa \& Araújo et al.,2014).

Outros aspectos que devem ser cruciais é a educação em saúde, portanto a não conscientização por parte do idoso da importância do seguimento da terapia pode refletir em piora na condição crônica. Assim Outro estudo realizado por Abreu et al., (2019) afirmam que os idosos tomavam os medicamentos somente quando apresentavam sintomas da condição crônica de saúde. Estes apresentaram menor nível de adesão do que aqueles que seguiam o tratamento de forma contínua. Outra explicação para a elevada taxa de não adesão é que muitos pacientes não compreendem a doença e o tratamento medicamentoso (Krieger, 2014).

A repercussão disso quanto à adesão ao tratamento é que dificulta no entendimento da patologia de base, os cuidados necessários, a tomada de medicamentos, dentre outras providências que impactam na qualidade de vida (Silva et al., 2018).

Concordo a explanação do autor de modo que os homens tem mais resistência em aderir o tratamento. o gênero masculino, faixa etária entre 20 e 59 anos, baixa classe econômica, etilismo, tempo curto de diagnóstico e a não procura pelo serviço de saúde para consultas de rotina foram os fatores que apresentaram associação com a não adesão ao tratamento medicamentoso da HAS (Magnabosco 2015).

Diante deste contexto, os profissionais de saúde responsáveis pelo acompanhamento dos idosos com HAS podem usar a escuta no planejamento ações resolutivas; educação em saúde; orientação sobre hábitos de vida saudáveis de forma clara, com o objetivo do autocuidado; e práticas humanizadas promotoras da empatia, pois facilitam a adesão, o controle pressórico e propiciam o sucesso do tratamento (Dias et al.,2016).

Os profissionais de saúde deveriam estabelecer uma relação dialogada e realizar ações educativas com os idosos para demonstrar a importância da mudança integral no estilo de vida, preservando seu empoderamento. Tais estratégias visam o controle da pressão arterial e redução de riscos de complicações, subsidiando uma assistência qualificada e integral dos idosos com HAS (Falcão, et al.,2018).

No dia a dia do enfermeiro, a consulta de enfermagem está ligada ao processo educativo e deve motivar a pessoa em relação aos cuidados necessários para a manutenção de sua saúde. Na prática, representa importante instrumento de estímulo à adesão às ações na Atenção Primária à Saúde e tem sido fundamental no acompanhamento de pessoas com pressão arterial limítrofe e portadoras de HAS, sensibilizando-as sobre a sua condição de saúde e pactuando com elas metas e planos de como seguir o cuidado (Costa \& Araújo et al., 2014). Assim, vale refletir que é necessário que haja abordagens inovadoras que promovam maior adesão ao tratamento hipertensivo, e que favorecem a mudança de comportamento.

\section{Considerações Finais}

Diante deste cenário pode-se perceber que as literaturas encontradas mostram que os fatores preditoras para a não adesão ao tratamento medicamentoso da HAS em idoso se dá pela falta de informações, baixa escolaridade, falta de apoio familiar. Portanto, estratégias que envolvam acolhimento, educação em saúde e mudança de comportamento podem favorecer um comportamento participativo que contribua para a adesão terapêutica de maneira adequada. 
Entretanto faz-se necessário que os profissionais de saúde reconheçam os principais fatores que contribuem para a não adesão do tratamento medicamentoso desta doença e assim identificar os grupos vulneráveis, pois é de grande valia para elaboração de estratégias que possam combater o tratamento deficiente e uma significativa adesão dos pacientes hipertensos, e assim contribuir para um controle pressórico mais eficiente.

Cabe ressaltar que a adesão ao tratamento medicamentoso não deve ser restrita às consultas médicas; antes, deve envolver a participação de uma equipe multidisciplinar, onde o enfermeiro possa atuar de forma integrada na abordagem da avaliação de risco, adoção de medidas de promoção da saúde e educação permanente, garantindo um atendimento qualificado aos usuários dos serviços de acompanhamento ambulatorial.

O estudo visa contribuir para uma melhor reflexão sobre atuação do enfermeiro na atenção básica para promover a mudança de comportamento e enfatizando sua importância em identificar os fatores comportamentais que podem interferir a não uso de medicamentos. Porém, há necessidade de maiores investigações para os futuros pesquisadores sobre a temática que ainda desafia a comunidade cientifica.

\section{Referências}

Abreu, D. P. G., Santos., S. S. C., Ilha, B. T. S., Martins, N. F. F., Varela, V. D. S.(2019).Fatores Comportamentais Associados à Adesão Medicamentosa Em Idosos Em Atendimento Ambulatorial. Revista de Enfermagem do Centro Oeste Mineiro,9. http://dx.doi.org/10.19175/recom.v9i0.3025

Barreto, M., Reiners, A. A. O., \& Marcon, S. S (2014). Conhecimento sobre hipertensão arterial e fatores associados à não adesão à farmacoterapia. Revista Latino-Americana de Enfermagem, 22(3), 491-498.\%

Bezerra, A. S. M., Lopes, J. L., \& Barros, A. L. B. L. (2014). Adesão de pacientes hipertensos ao tratamento medicamentoso. Revista Brasileira de Enfermagem, 67(4), 550-555.

Borba, A. K. D. O. T., Marques, A. P. D. O., Ramos, V. P., Leal, M. C. C., Arruda I. K. G. D., \& Ramos, R. S. P. D. S. (2018).Fatores associados à adesão terapêutica em idosos diabéticos assistidos na atenção primária de saúde. Ciênc Saúde Coletiva 23(3),953-61. 10.1590/1413-81232018233.03722016

Brasil, Sociedade Brasileira de Hipertensão, Sociedade Brasileira de Nefrologia. (2017).VI Diretrizes Brasileiras de Hipertensão. Arq Bras Cardiol,95(1),1-51.

Cofen Conselho Federal de Enfermagem Resolução Cofen 311/2007. Aprova a Reformulação do Código de Ética dos Profissionais de Enfermagem 2007.

Costa, Y. F., \& Araújo, O. C.(2014).O papel educativo do enfermeiro na adesão ao tratamento da Hipertensão Arterial Sistêmica: revisão integrativa da literatura: integrative literature review $O$ Mundo da Saúde, São Paulo 38,(4) P.473-4.

Cunha, P. L. P., Cunha, C. S. D. \& Alves, P. F. (2014).Manual Revisão Bibliográfica Sistemática Integrativa. a pesquisa baseada em evidências

Dias, E. G., Souza, E. L. S., \& Mishima, S. M. (2016). Contribuições da enfermagem na adesão ao tratamento da hipertensão arterial: uma revisão integrativa da literatura brasileira. Revista Epidemiologia Controle Infecção 6(3),138-44.

Falcão, A. S., Silva, M. G. C., Rodrigues, A. F., Junior, M. S. R., Silva, F. R. S., Sousa, A. S. J. (2018) Estilo de vida e adesão ao tratamento de hipertensão arterial sistêmica em homens idosos Rev Bras Promoção Saúde, 31(2), 1-10.

Gewehr, D. M., Bandeira, V. A. C., Gelatti, G. T., Colet, C. F., Oliveira, K. R. (2018). Adesão ao tratamento farmacológico da hipertensão arterial na Atenção Primária à Saúde, Saúde Debate 42(116),179-190

http://biblioteca.cofen.gov.br/wp-content/uploads/2019/06/manual_revisao_bibliografica-sistematica-integrativa.pdf

Instituto Brasileiro de Geografia e Estatística. Censo demográfico 2006. ftp://ftp.ibge.gov.br/PNS/2006/pns2006.pdf

Jannuzzi, F. F., Rodrigues, R. C. M., Cornélio, M. E., São-João, T. M., \& Gallani, M. C. B. J. (2014) Beliefs related to adherence to oral antidiabetico treatmenta ccording to the Theory of Planned Behavior. Rev Latino-Am Enfermagem 22(4) 529-37. https://dx.doi: 10.1590/01041169.3578.2448 .

Jesus, N. S., Nogueira, A. R., Pachu, C. O., Luiz, R. R., Oliveira, G. M. M. (2016). Adesão ao Tratamento e Controle da Pressão Arterial após Participação no ReHOT. Arquivos Brasileiros de Cardiologia, 107(5), 437-445. https://dx.doi.org/10.5935/abc.20160165

lei no 10.741, de $1^{\text {o }}$ de outubro de 2003.(2003) Dispõe sobre o Estatuto do Idoso e dá outras providências. http://www.planalto.gov.br/ccivil_03/leis/2003/110.741.htm

Magnabosco, P., Teraoka, E. C., Oliveira, E. M., Felipe, E. A., Freitas, D., Marchi-Alves, L. M. (2015). Comparative analysis of non-adherence to medication treatment for systemic arterial hypertension in urban and rural populations. Revista Latino-Americana de Enfermagem,23(1), 2027. https://dx.doi.org/10.1590/0104-1169.0144.2520

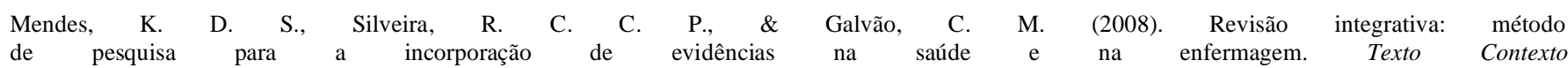
Enferm., 17(4), 758.764 
Research, Society and Development, v. 10, n. 1, e50510111863, 2021

(CC BY 4.0) | ISSN 2525-3409 | DOI: http://dx.doi.org/10.33448/rsd-v10i1.11863

Ministério da Saúde. Secretaria de Atenção à Saúde. Departamento de Atenção Básica. (2013). Estratégias para o cuidado da pessoa com doença crônica:

$\begin{array}{llccccc}\text { hipertensão } & \text { arterial } & \text { sistêmica. } & \text { Ministério } & \text { da } & \text { Saúde, } & \text { Secretaria } \\ \text { Saúde, Departamento de Atenção Básica. Cadernos de Atenção Básica, Brasília https://aps.saude.gov.br/biblioteca/visualizar/MTIxNA }\end{array}$

Pinheiro, F. M., Espirito Santo, F. H., Sousa, R. M., Silva, J. D., \& Santana,R. F. (2018).adesão terapêutica em idosos hipertensos: revisão integrativa. Revista de Enfermagem do Centro-Oeste Mineiro,(8) https://dx doi: 10.19175/recom.v8i0.1938

Silva, D. F. D., Araújo, N. C. D. S., \& Campos, E. A. D.(2018).Perfil dos pacientes hipertensos e diabéticos atendidos na Atenção Básica. Revista de Enfermagem da Faciplac 2(2)

Silveira, A. G., \& Silva, D. A. (2020). Sobrecarga dos familiares no cuidado de pacientes com demência senil: uma revisão integrativa. Research, Society and Development, 9 (6), e179963671. https://doi.org/10.33448/rsd-v9i6.3671

Torres, G. M. C., Figueiredo, I. D. T., Cândido, J. A. B., Morais, A. P. P., \& Almeida, M. I. (2018). The use of soft technologies in the care of hypertensive patients in Family Health Strategy. Escola Anna Nery, 22(3), e20170169. Epub June 11, 2018.https://doi.org/10.1590/2177-9465-ean-2017-0169 